\title{
RESPOSTAS FALSO-POSITIVAS NA TRIAGEM AUDITIVA NEONATAL UNIVERSAL: POSSÍVEIS CAUSAS
}

\author{
False-positive results in newborn universal hearing screening: \\ possible causes
}

\author{
Maria Cristina Silva Simonek ${ }^{(1)}$, Marisa Frasson de Azevedo ${ }^{(2)}$
}

\begin{abstract}
RESUMO
Objetivo: avaliar a ocorrência de respostas falso-positivas na Triagem Auditiva dentro de uma maternidade particular, suas possíveis causas e soluções. Método: foram avaliados 1.110 recém-nascidos eutróficos entre 6 e 48 horas de vida no berçário, com o analisador de Emissões Otoacústicas Evocadas Transientes (EOATE) modelo Echochek da llodynamics Ltd. Na ausência de resposta, foi realizada a manipulação do meato acústico externo (Manobra Facilitadora) e colhido novo resultado. Os que falharam foram retestados em 15 dias. Resultados: 50,09\% dos RN falhou na primeira tentativa. Após a manobra facilitadora, $24,41 \%$ continuou falhando, mas demonstraram EOATE presentes no reteste. O tempo médio de internação foi de $42,27 \mathrm{~h}$, sendo $93,42 \%$ oriundos de cesareana. Pertenciam a convênios particulares padrão enfermaria $98 \%$ e $2 \%$ quarto individual. A idade média do grupo que passou foi de $24,14 \mathrm{~h}( \pm 10,21)$ e a do grupo que falhou 19,19 horas $( \pm 8,43)$. Possuíam menos de 24 horas de vida no momento do teste $66,12 \%$ dos $\mathrm{RN}$, foi constatado vérnix obliterante em 4.9\%. Conclusão: face a alta precoce hospitalar, é necessário que o Fonoaudiólogo realize o teste antes das 48 horas de vida do RN. Desta forma, além das causas biológicas inerentes a faixa etária, detectou-se um problema estrutural de administração hospitalar, que independe da habilidade ou experiência do Fonoaudiólogo. A Manobra Facilitadora é altamente recomendada.
\end{abstract}

DESCRITORES: Audição; Recém-Nascido; Surdez; Alta do Paciente

\section{INTRODUÇÃO}

Nos programas de Triagem Auditiva Neonatal Universal (TANU) a quantidade de recém-nascidos (RN) com audição normal e emissões otoacústicas (EOA) ausentes, quando testados nas primeiras 48 horas após o nascimento, é uma questão complexa. Altos índices de respostas falso-positivas

(1) Fonoaudióloga do Instituto Nacional de Educação de Surdos (INES), Rio de Janeiro, Rj- Brasil.; Mestre em Ciências dos Distúrbios da Comunicação Humana pela Universidade Federal de São Paulo - Escola Paulista de Medicina(UNIFESP)

(2) Fonoaudióloga da Universidade Federal de São Paulo Escola Paulista de Medicina, Departamento de Distúrbios da Comunicação Humana, Programa de Pós-graduação em Distúrbios da Comunicação Humana: Campo Fonoaudiológico, São Paulo- SP, Brasil. Professora Doutora em Ciências dos Distúrbios da Comunicação Humana pela Universidade Federal de São Paulo - Escola Paulista de Medicina

Conflito de interesses: inexistente comprometem a qualidade dos programas de TANU ${ }^{1}$ e aumentam desnecessariamente a ansiedade dos pais ${ }^{2}$.

No Brasil, o Comitê Brasileiro sobre Perdas Auditivas na Infância (CBPAI) ${ }^{3}$ recomendou que o índice não deveria exceder $3 \%$ e a taxa de referenciamento para avaliação audiológica completa póstriagem não poderia ultrapassar 4\%, valores semeIhantes aos da Academia Americana de Pediatria e do Comitê multidisplinar de Saúde Auditiva ${ }^{4}$. Ambas as recomendações não levam em conta, a multiplicidade de infra-estruturas hospitalares existentes. Na realidade, os dados do primeiro grande programa de TANU realizado em Rhode IslandUSA ${ }^{5}$ apontavam 27\% de falso-positivo. Dessa forma, as primeiras recomendações sugeriam a realização do exame de EOA em RN de alto e baixo riscos e uma segunda etapa da triagem utilizando o Potencial Evocado Auditivo de Tronco Encefálico (PEATE) ${ }^{6}$ nos RN que falhassem, ambos antes da alta hospitalar. Entretanto, a facilidade de aplicação 
fez das EOA o instrumento mais utilizado na realização da TA até o presente momento. Na Itália, o protocolo de TA utilizando somente EOA é aplicado por $92,8 \%$ dos programas baseados dentro das maternidades, contrapondo com o modelo EOATE/ PEATE utilizado apenas por $2 \%$ dos serviços ${ }^{7,8}$.

A preocupação com as falhas aumentou face à tendência hospitalar mundial de "alta precoce" nas maternidades, sugerindo que nos hospitais com alta antes das 24 horas de vida, a implantação da TANU com EOA seria inviável ${ }^{9}$.

O objetivo deste estudo foi, portanto, verificar o índice de respostas falso-positivas e suas possíveis causas, dentro de um programa préviamente estabelecido numa Maternidade, sejam elas clínicas dos RN ou operacionais e, a partir do conhecimento das causas, propor soluções para minimizar o índice de falhas.

\section{MÉTODO}

Foram estudados 1.110 RN eutróficos, sem indicadores de risco para a surdez, conforme recomendação do $\mathrm{JClH}{ }^{10}$, sendo $48,83 \%$ do sexo feminino e $50,99 \%$ do sexo masculino, na faixa etária entre 6 e 48 horas de vida. Os dados foram colhidos em programas de TANU de três maternidades particulares do Estado do Rio de Janeiro. O padrão de assistência hospitalar utilizava convênios médicos tipo standard com direito à habitação em quarto coletivo.

A TA foi realizada por fonoaudiólogos experientes (>5.000 exames de prática), diariamente no próprio berçário, utilizando o equipamento modelo Echocheck da llodynamics Ltd. Adotou-se como critério de "passa" na triagem, a presença de EOAET em ambas as orelhas na relação sinal ruído de 6 dB. Como critério de exclusão, estabeleceu-se a ausência de Reflexo Cócleo Palpebral (RCP), RN fora do estado adequado para o teste e os que, no reteste, não apresentaram EOA. Nesses programas, era rotina avaliar a presença do RCP.

O protocolo clínico metodológico utilizado nessa população seguiu o recomendado pelo $\mathrm{CBPAI}^{3}$ e pelo COMUSA ${ }^{4}$, o que possibilitou a utilização do exame de EOA como procedimento único de triagem.

Em caso de ausência de resposta, foi realizada a Manobra Auricular Facilitadora (MAF), reinserida a sonda e colhido novo resultado. Os RN que continuaram falhando depois da aplicação da MAF foram encaminhados para reteste em 15 dias. No reteste, ambas as orelhas foram reavaliadas, mesmo que apenas uma delas tivesse falhado na TA inicial, conforme recomendação do $\mathrm{JCIH}^{10}$. Após o reteste, foram estabelecidos os índices de falso-positivo.
A MAF consistiu na manipulação do meato acústico externo (MAE) com a realização de movimentos circulares com o dedo indicador sobre o tragus no sentido anti-horário e o deslocamento simultâneo do pavilhão no sentido póstero-inferior, auxiliado pelo dedo polegar. A população de $\mathrm{RN}$ foi submetida ao teste sem realizar a retirada do vérnix do meato acústico externo.

Para evitar a evasão no reteste ${ }^{11}$ foram realizados os seguintes procedimentos: aconselhamento, encaminhamento escrito com data e hora do retorno, telefonema e oferecimento de pacotes de fralda.

Os dados clínicos obtidos de cada RN, sexo, idade gestacional, correlação peso/idade, ocorrência de vérnix, horas de vida do RN no momento do teste e índice de falha por orelha foram analisados e comparados às características operacionais da entidade hospitalar, tais como padrão do convênio médico, alojamento, tempo de internação, tipo de parto, tipo de banho e local destinado à realização da TA, uma vez que tais fatores podem influenciar a rotina da TANU.

Este trabalho foi aprovado pelo Comitê de Ética em Pesquisa (CEP), sob o protocolo no 0367/05. Todas as mães dos RN envolvidos consentiram a realização desta pesquisa e a divulgação dos resultados conforme resolução 196/96 do Ministério da Saúde.

A análise estatística dos dados foi feita através do teste de hipótese e de significância, sendo considerado como nível de significância o valor de 0,05 ou $5 \%$.

Quando constatadas diferenças estatisticamente significantes, foi realizado o cálculo das Razões de Chance (Odds Ratio) a fim de ilustrar de maneira mais clara a relação entre a variável dependente e as variáveis independentes. Para verificar se existia diferença estatisticamente significante das horas de vida (considerada como variável continua) entre aqueles que passam e aqueles que falham na TANU foi utilizado um modelo de ANOVA (Análise de Variância).

\section{RESULTADOS}

Dos 1.110 RN da amostra, 556 (50,09\%) falharam na primeira tentativa de TA. Foi realizado neste grupo a MAF com reinserção da sonda e coletado novo resultado. Desses, 285 (51,26\%) passaram e 271 (48,74\%) continuaram falhando e foram encaminhados para reteste pós-alta. Os 271 RN que falharam na triagem pré-alta demonstraram EOA presentes no reteste em 15 dias. Portanto, a ocorrência de respostas falso-positivas neste estudo foi de $24,41 \%$. 
A necessidade de realização da MAF variou de acordo com o número de horas de vida, conforme demonstrado na Tabela 1.

Tabela 1 - Necessidade de realização da MAF de acordo com as horas de vida/teste (razão de chance)

\begin{tabular}{lcc}
\hline Grupos etários & RC (OD) & RC (OE) \\
\hline$<12 x>36<48 \mathrm{~h}$ & 5,0 & 5,0 \\
$>12<24 \mathrm{x}>36<48 \mathrm{~h}$ & 3,0 & 3,0 \\
$>24<36 \mathrm{x}>36<48 \mathrm{~h}$ & Igual & Igual \\
\hline
\end{tabular}

$\mathrm{RC}=$ Razão de chances

Em relação ao sexo, foi constatada diferença estatisticamente significante $(p<0,05)$ entre os resultados da TA para os indivíduos dos sexos feminino e masculino. A chance de um RN do sexo masculino falhar é 1,5 vezes maior do que a chance de um $\mathrm{RN}$ do sexo feminino.

A diferença para os sexos mostrou-se consistente também quando a análise foi realizada separadamente por orelha. $\mathrm{Na} O D$, a chance de um $\mathrm{RN}$ do sexo masculino falhar é duas vezes maior do que a do sexo feminino.

$\mathrm{Na} \mathrm{OE}$, a proporção de recém-nascidos do sexo masculino que falhou é maior do que a proporção do feminino. A chance de um $\mathrm{RN}$ do sexo masculino falhar na OE é 1,5 vezes maior que a chance de um $\mathrm{RN}$ do sexo feminino.

Em relação à modalidade de falha, ocorreu uma concentração na modalidade bilateral $(84,87 \%)$. Dentro da modalidade de falha unilateral, a OE foi predominante, apresentando $82,93 \%$ de falha.

O número de horas de vida do RN no momento do teste demonstrou diferença estatisticamente significante entre os grupos de faixa etária e o índice de falha na TANU (Tabela 2).

Tabela 2 - Ocorrência de falha na TA em relação a horas de vida do RN

\begin{tabular}{|c|c|c|c|c|c|c|}
\hline \multirow{3}{*}{ Horas de Vida } & \multicolumn{4}{|c|}{ Índice de Falha } & \multirow{2}{*}{\multicolumn{2}{|c|}{ Total }} \\
\hline & \multicolumn{2}{|c|}{ Passou } & \multicolumn{2}{|c|}{ Falhou } & & \\
\hline & $\mathbf{N}$ & $\%$ & $\mathbf{n}$ & $\%$ & $\mathbf{N}$ & $\%$ \\
\hline Sem Informação & 1 & 100 & 0 & 0,00 & 1 & 100 \\
\hline$\leq 12$ & 86 & 65,15 & 46 & 34,85 & 132 & 100 \\
\hline$>12 \leq 24$ & 423 & 70,27 & 179 & 29,73 & 602 & 100 \\
\hline$>24 \leq 36$ & 214 & 87,7 & 30 & 12,3 & 244 & 100 \\
\hline$>36 \leq 48$ & 115 & 87,79 & 16 & 12,21 & 131 & 100 \\
\hline Total & 839 & & 271 & & 1110 & \\
\hline
\end{tabular}

$p<0,0001$

Os RN que passaram na TA possuíam, em média, cinco horas de vida a mais do que os que falhavam $(p<0,0001)$. A razão de chances de um RN falhar de acordo com as horas de vida/teste é demonstrada na Tabela 3.

Neste estudo, realizaram a TA antes das 24 horas de vida, $66,12 \%$ dos RN, tendo sido constatada a presença de vérnix obliterante em $84 \mathrm{RN}$ (7,57\%). Os RN abaixo de 12 horas apresentaram 6 vezes mais chance de vérnix do que os RN acima de 24 horas.

O tempo médio de internação das mães a partir do parto e dos neonatos até a alta foi de 42,27 horas. $O$ índice de encaminhamento para avaliação audiológica completa foi igual a zero.

Não foi encontrada diferença estatisticamente significante $(p>0,05)$ nos índices de passa/falha entre as variáveis: tipo de parto, trabalho de parto, idade gestacional, peso e realização da TA pré ou pós-banho. O elevado índice de cesarianas $(93,42 \%)$ inviabilizou a análise comparativa da ocorrência de falso-positivos entre os partos cesárea e normal.

Tabela 3 - Probabilidade de falha na TANU de acordo com horas de vida/teste (razão de chance)

\begin{tabular}{lc}
\hline Grupos etários & RC \\
\hline$<12 x>36<48 h$ & 4,0 \\
$>12<24 x>36<48 h$ & 4,0 \\
$>24<36 x>36<48 h$ & Igual \\
$<12 x>12<24$ & Igual \\
\hline
\end{tabular}

$\mathrm{RC}=$ Razão de chances 


\section{DISCUSSÃO}

O tempo de permanência hospitalar vem decrescendo em vários países e esta tendência tem sido incorporada no Brasil, particularmente na Obstetrícia. A Academia Americana de Pediatria em conjunto com o Colégio Americano de Obstetrícia e Ginecologia (ACOG) recomendaram que o tempo médio de internação para parto normal não complicado deveria ser 48 horas e de $96 \mathrm{~h}$ para cesariana $^{12}$. No nosso meio não existe consenso oficial, havendo referência para a portaria número 1016 do Ministério da Saúde que recomenda que as altas não devem ser dadas antes das 48 horas. Os entusiastas da alta precoce afirmam que ela é segura e vantajosa do ponto de vista médico, social e econômico, por reduzir o número de infecções hospitalares, satisfazer a vontade dos pais com menor tempo de internação e diminuição de custos hospitalares.

Entretanto, as desvantagens para a execução de um programa de triagem auditiva neonatal, são diversas, como constatatado nesse estudo. Em consequência de um tempo de internação reduzido $(42,27 \mathrm{~h})$, o teste foi aplicado antes das 24 horas de vida em mais da metade dos RN (66.12\%). A alta precoce impede ainda que seja feita uma nova tentativa antes da alta hospitalar.

Nesse estudo, o fato de $66,12 \%$ dos RN possuir menos de 24 horas pode justificar os índices obtidos. Estes dados concordam com os descritos na literatura, que apontam o número de horas de vida do RN no momento do teste como o principal preditor de falha na TANU ${ }^{5,13-15}$. A realização do teste após as primeiras 24 horas de vida parece ser um determinante importante para que um $\mathrm{RN}$ ouvinte não falhe na TA

A manipulação do MAE, neste estudo denominado de Manobra -Facilitadora (MAF) demonstrou ser altamente satisfatória e recomendável. Observou-se que os resultados da ocorrência de falha na TA antes da realização da MAF $(50,09 \%)$ concordavam com os encontrados na literatura pesquisada quando avaliaram RN com menos de 48 horas de vida e não realizavam a retirada do vérnix do MAE ${ }^{13,16}$. Em contrapartida, discordam dos $20 \%$ previstos quando a TA é realizada nas primeiras 24 horas $^{3}$.

Após a MAF os achados $(24,41 \%)$ foram semeIhantes aos obtidos em pesquisas realizadas em RN maiores que 48 horas ${ }^{5,14,17}$ e próximo ao previsto quando a TA é realizada nas primeiras horas de vida do $\mathrm{RN}^{3,16}$. Estudos onde o critério de "passa" considera apenas uma orelha ${ }^{18}$ também obtiveram índices inferiores (12,3\%) a esse estudo.
De maneira geral, é possível inferir que o índice de falha está associado ao número de horas de vida do RN no momento do teste. Entretanto, a MAF pode diminuir esses índices. Os trabalhos com índices de falha inferiores a este estudo só foram obtidos nas pesquisas que realizaram a limpeza do vérnix no $M A E^{13}$, ou naqueles que estudaram sómente $R N$ acima de 24 horas e destaforma concluíram que não houve diferença com relação a faixa etária. ${ }^{19}$

Outros estudos na literatura apontam a associação do teste de EOATE com o PEATE ${ }^{6,20-22}$ ou ainda a realização de uma segunda tentativa de obtenção das EOA mais próximo à alta ${ }^{20,23}$ como formas de minimizar as falhas. Porém, a redução do tempo de internação, inferior a $96 \mathrm{~h}$ para cesárea e $48 \mathrm{~h}$ para parto normal não permitiu outras estratégias.

O baixo índice de evasão no reteste está possívelmente relacionado a estratégias utilizadas e se contrapõe aos $10 \%$ encontrado em outros países em desenvolvimento e alto índice de natalidade. ${ }^{11}$

$\mathrm{O}$ fato dos $\mathrm{RN}$ do sexo masculino apresentarem maior número de falhas é descrito na literatura e pode ser explicado porque no sexo feminino as amplitudes das EOA são maiores. ${ }^{24-26}$ Nessa faixa etária, os níveis de ruído são exacerbados pela presença de líquido amniótico na orelha média. Portanto, a associação do nível de ruído com a baixa amplitude levaria a um maior índice de falhas justificando os achados da presente pesquisa.

A concentração de falhas na modalidade bilateral $(84,87 \%)$ pode estar associada ao fato que as mães colocavam os RN aleatoriamente em decúbito lateral direito ou esquerdo no alojamento conjunto, independente da recomendação para o esvaziamento gástrico pós-mamada à direita.

Dentro da modalidade de falha unilateral, a maior concentração de falhas na orelha esquerda é citada na literatura ${ }^{24}$ e pode ser justificada pela constatação das baixas amplitudes das EOA nesse lado em comparação com a orelha do lado direito na população de neonatos.

A presença de vérnix caseoso obliterando o MAE é apontada na literatura como uma das principais causas de alterações no exame ${ }^{13,16}$. Neste estudo, a presença de vérnix obliterante em 4,9\% das orelhas, é menor que o encontrado na literatura consultada que refere $13 \%$ totalmente obstruídas ${ }^{13}$ e $43 \%$ entre parcial e total. A diferença pode estar associada ao método de inspeção do MAE, neste estudo um Fonoaudiólogo com otoscópio comum e nos citados ${ }^{13}$ um Médico Otologista com um otoscópio pneumático. Outros estudos apontam uma diminuição das falhas de $41,5 \%$ para $4 \%$ em RN entre 5 e 48 horas de vida utilizando o método de Sucção para retirada do vérnix ${ }^{13}$. 
Este estudo tenta demonstrar que o vérnix, só altera significativamente a TA, quando é realizada antes das 24 horas, desta forma se contrapõe as afirmações do Comitê multidisplinar de Saúde Auditiva ${ }^{4}$, que cita que "devido a ocorrência de falsopositivos pela presença de vérnix nos primeiros dias de vida", não é questão de dias e sim de horas após o nascimento.

Em todos os RN foi utilizada a oliva T4.5C, projetada para RN pequenos com MAE de 4-5mm. Em contrapartida na população de $\mathrm{RN}$ ingleses, o recomendado foi o modelo T5.5B, indicado para MAE acima de $5,5 \mathrm{~mm}^{18}$, sugerindo que podem existir variações em diferentes povos, sendo o Brasileiro menor que o Europeu. Não foi constatada diferença estatisticamente significante com relação ao peso e a idade gestacional dos RN na TA.

A correlação entre o tipo de parto e a falha na TA não foi detectada, entretanto, foram constados dados alarmantes nessa população, 93,42\% para as Cesarianas, índices muito acima dos 15\% propostos pela Organização Mundial da Saúde. O Brasil é o segundo colocado em realização de partos cesárea do mundo ${ }^{27}$
Os convênios médicos particulares são hoje responsáveis pelo atendimento obstétrico de 14 milhões de brasileiras com idade entre 10 e 49 anos ${ }^{27}$, entretanto, no Rio de Janeiro, somente as maternidades públicas estão obrigadas a realizar a TA ${ }^{28}$. Apesar disso, durante a vigência desse estudo, a TANU atingiu 95\% dos RN nesses hospitais ${ }^{3}$.

\section{CONCLUSÃO}

O programa avaliado apresentou um índice de resposta falso-positiva de $24,41 \%$ para um tempo médio de permanência hospitalar de $42,27 \mathrm{~h}$. Não é possível dissociar esses fatores, porque deles depende o número de horas do RN no momento do teste. As causas do falso-positivo são a tendência à alta precoce hospitalar, associada ao baixo número de horas de vida do RN. As possíveis soluções, para minimizar esses índices, incluem a aplicação generalizada da Manobra Auricular Facilitadora nas primeiras horas de vida e a decisão em conjunto com os profissionais que prestam assistência ao $\mathrm{RN}$, em concordância com a família, sobre o momento adequado para a alta.

\begin{abstract}
Purpose: to investigate the rate of false positive responses in a hospital - based newborn hearing screening program (NHS), their possible causes and solutions. Method: during the period we evaluated 1,110 regular nursery newborns, age 6 to 48 hours with Transient Evoked Otoacoustic Emissions (TEOAEs), Echocheck, llodynamics Ltd model., at the nurseries, before discharge. In case of absence of response, the ear canal was manipulated. Infants that failed the screening were retested fifteen days after birth. Results: $50.09 \%$ failed the first trial, then the ear canal was manipulated, and it decreased to $24.41 \%$. This group showed normal TEOAEs at the retest time; Mean time of hospital discharge was $42,27 \mathrm{~h}$, thought $93 \%$ cesarian delivery; $98 \%$ double room and $2 \%$ single room status. The mean age of the "pass" group was $24.14 \mathrm{~h}( \pm 10.21)$ and the "fail" group 19.19 hours $( \pm 8.43) .66 .12 \%$ had less than 24 hours on the moment of the test. $4.9 \%$ were occluded with vernix. Conclusion: hospital trend toward "early discharge" after routine deliveries forces the audiologist to carry out the screening before 48 hours. High number of false-positives in NHS, must be seen as a Hospital Administrative Problem not only as a biologic cause, like the "vernix caseosum", and it is not related to the professional experience. Ear manipulation is strongly recommended.
\end{abstract}

KEYWORDS: Hearing; Infant, Newborn; Deafness; Patient Discharge 


\section{REFERÊNCIAS}

1. Bohomol E, D'Innocenzo M, Kowal I, Cunha O. Indicadores de qualidade conceitos e sistemas de monitoramento. Artigo de Revisão. Rev Centro Universitário São Camilo. 2005; 11(2):75-81.

2. Simonek MC. Avaliação audiológica. Sociedade de Pediatria do Rio de Janeiro (SOPERJ). In: Lanzelotte $\mathrm{V}$. Manual de atenção integral ao desenvolvimento e reabilitação. Rio de Janeiro: Revinter; 2007; 5: 50-70.

3. Comitê Brasileiro Sobre Perdas Auditivas na Infância (CBPAl). Recomendação 01/99 do Comitê Brasileiro sobre perdas auditivas na Infância. J Conselho Federal de Fonoaudiologia. 2000; 5:3-7.

4. Comitê Multidisciplinar em Saúde Auditiva Assunto: Parecer sobre TANU. São Paulo. [acesso em 20 maio 2009] Disponível em: URL: http://www. audiologiabrasil.org.br/pdf/COMUSA 5. White KR, Vohr BR, Behrens TR. Universal newborn hearing screening using transient evoked otoacoustic emissions: results of the Rhode Island Assessment Project. Semin Hear. 1993; 14:18-29.

6. Ito-Orejas JI, Ramirez B, Morais D, Almaraz A, Fernandez-Calvo JL. Comparison of two-step transient evoked otoacoustic emissions (TEOAE) and automated auditory brainstem response (AABR) for universal newborn hearing screening programs. Int J Pediatr Otorhinolayngol. 2008; 72:1193-201.

7. Bubbico L, Bartolucci MA, Broglio D. The newborn hearing screening in italy. It $\mathrm{J}$ Pediatr. 2005; 31:290-2.

8. De Capua B, Costantini D, Martufi C, Latini G, Gentile M, De Felice C. Universal neonatal hearing screening: The Siena (Italy) experience on 19.700 newborns. Early Hum Dev. 2007; 83(9):601-6.

9. Grill E, Uus K, Hessel F, Davies L, Taylor RS, Wasem J. Neonatal hearing screening: modeling cost and effectiveness of hospital and communitybased screening. BMC Health Serv Res. 2006; 6:14. 10. Joint Committee on Infant Hearing. Year 2007 position statement: principles and guidelines for early hearing detection and intervention programs. Pediatrics. 2007; 120:898-921.

11. Olusanya BO. Folow-up default in a hospitalbased universal newborn hearing screening programme in a low-income country. Childcare health Dev. 2009; 35(2):190-8.

12. Sociedade Brasileira de Pediatria (SBP) Assunto: Tempo de Permanência Hospitalar do recém-nascido. São Paulo. [homepage na internet]. 2004 [acesso em 1 ago 2006] Disponível em: http:// www.sbp.com.br

13. Doyle KJ, Fujikawa S, Rogers P, Newman E. External and middle ear effects on infant hearing screening test results. Otolaryngol Head Neck Surg. 2000; 122(4):477-81.

14. Torrico P, Gómez C, López-Ríos J, De Cáceres MC, Trinidad G, Serrano M. Age influence in otoacoustic emissions for hearing loss screening in infants. Acta Otorrinolaringol Esp. 2004; 55:153-9. 15. Asma A, Wan FWH, Almyzan A, Han YS, Jamilah AG, Roslin S, et al. Benefit and pitfalls of newborn hearing screening. Med $\mathrm{J}$ Malaysia 2008;63 (4):293-7

16.Dhawan R, Mathur N. An alternative strategy for UHS in tertiary hospitals, within a developing country, using transient evoked oto-acoustic emissions and brainstem evoked response audiometry. The J Laryngol \& Otology. 2007; 121(7): 639-43.

17. Jakubikova J,Kabatova Z, Pavlovcinova G, Profant $M$, et al. Newborn hearing screening and strategy for early detection. Int $\mathrm{J}$ pediatr Otorhinolaringol 2009; 73(4):607-12.

18. Albuquerque $\mathrm{W}, \mathrm{Kemp}$ DT. The feasibility of hospital based universal newborn hearing screening in the United Kingdom. Scand Audiol. 2001; 30(53):22-8.

19. Melo ADP, Duarte JL, Alvarenga KF, Agostinho PRS, Beviláqua MC, Martinez MAN. Influencia do tempo de vida na pesquisa das emissões otoacústicas transientes em recém-nascidos. Distúrb.Comun 2007; 19 (3): 357-64.

20. Monteiro PC. Custo $x$ Efetividade da triagem auditiva neonatal em uma maternidade de São Paulo. [dissertação] São Paulo (SP): Pontifícia Universidade Católica de São Paulo; 2008. 149p.

21.Benito-Orejas J, Ramirez B, Morais D, Almaraz A, Fernandez-calvo JL. Comparison of two-step transient evoked otoacoustic emissions (TEOAE) and automated auditory brainstem response (AABR) for universal newborn hearing screening programs. Int J Pediatr Otorhinolaryngol 2008; 72 (8): 1193-201.

22. Jardim IS, Matas CG, Carvallo RMM. Emissões Otoacústicas Evocadas por estímulos transientes e potenciais auditivos de tronco encefálico automático na triagem auditiva neonatal. Eintein 2008; 6(3): 253-61

23.Pastorino GP, Sergi P, Mastrangelo M, Ravazzani P, Tognola G, Parazzini M, et al. The Milan Project: a newborn hearing screening programme. Acta Pediatr. 2005; 94(4):458-63.

24. Durante AS, Cavallo RMM, Costa FS, Soares JC. Características das emissões otoacusticas por transientes em programa de triagem auditiva neonatal. Pró-fono R.Atual.Cient.2005; 17 (2):133-140. 25. Soares JC, Carvallo RMM. Emissões otoacústicas evocadas por estímulo "tone burst" em neonatos. Braz. J. Otorhinolaryngol.2009; 75(3): 441-8. 
26. Sininger $\mathrm{Y}$, Cone B. Comment on "Ear asymmetries in middle-ear, cochlear and brainstem responses in human infants". J Acoust Soc Am 2008;124(3): 1401-3.

27. Zorzetto R. Estudo publicado na revista Lancet mostra que cesariana desnecessária coloca em risco a vida da mulher e do bebê. Rev Pesq Fapesp-Ciência e Tecnologia. 2006; 124: 3-5.

28. Tochetto TM, Vieira EP. Legislação brasileira sobre triagem auditiva neonatal. São Paulo: PróFono; 2006.

DOI: 10.1590/S1516-18462010005000076

RECEBIDO EM: 27/11/2009

ACEITO EM: 27/04/2010

Endereço para correspondência:

Maria Cristina Silva Simonek

Rua do Catete, 227 apto. 304, Bairro Catete

Rio de Janeiro - RJ

CEP: 22220-001

E-mail: surdez@surdez.com.br

Rev. CEFAC. 2011 Mar-Abr; 13(2):292-298 